SLAC-PUB-10566

hep-ph/0407039

July 2004

\title{
Markov Chain Monte Carlo Exploration of Minimal Supergravity with Implications for Dark Matter
}

\author{
Edward A. Baltz \\ KIPAC \\ SLAC MS 29 \\ 2575 Sand Hill Road \\ Menlo Park, CA 94025 \\ E-mail: eabaltz@slac.stanford.edu \\ Paolo Gondolo \\ Physics Department \\ University of Utah \\ 115 S 1400 E, Suite 201 \\ Salt Lake City, UT 84112 \\ E-mail: paolo@physics.utah.edu
}

\begin{abstract}
We explore the full parameter space of Minimal Supergravity (mSUGRA), allowing all four continuous parameters (the scalar mass $m_{0}$, the gaugino mass $m_{1 / 2}$, the trilinear coupling $A_{0}$, and the ratio of Higgs vacuum expectation values $\tan \beta$ ) to vary freely. We apply current accelerator constraints on sparticle and Higgs masses, and on the $b \rightarrow s \gamma$ branching ratio, and discuss the impact of the constraints on $g_{\mu}-2$. To study dark matter, we apply the WMAP constraint on the cold dark matter density. We develop Markov Chain Monte Carlo (MCMC) techniques to explore the parameter regions consistent with WMAP, finding them to be considerably superior to previously used methods for exploring supersymmetric parameter spaces. Finally, we study the reach of current and future direct detection experiments in light of the WMAP constraint.
\end{abstract}

KEYWords: Supersymmetry Phenomenology, Dark Matter.

Work Supported in part by the Department of Energy Contract DE-AC03-76SF00515

Stanford Linear Accelerator Center, Stanford University, Stanford, CA 94309 


\section{Introduction}

Two of the most interesting open questions in physics, the stabilization of the gauge hierarchy and the nature of the dark matter, naively appear to be related in that both point to new physics at the weak scale $\sim 100 \mathrm{GeV}$. Supersymmetric theories are an explicit realization of this relationship. The gauge hierarchy (in particular the Higgs mass) is stabilized by the interplay between fermionic and bosonic radiative corrections. Dark matter appears trivially with the imposition of R-parity conservation, which stabilizes the lightest superpartner (LSP). The relic density of stable particles with masses $\sim 100 \mathrm{GeV}$ undergoing thermal freeze-out in the early universe is generically found to be of the observed order.

Supersymmetric extensions to the Standard Model are plagued by a lack of predictiveness due to the large number of undetermined parameters. Even assumptions that drastically reduce the parameter space leave enough parameters that exhaustive study is difficult. In this paper we apply the well-known (in other fields) techniques of Markov Chain Monte Carlo (MCMC) to explore the parameter space of Minimal Supergravity (mSUGRA), a simple and widely studied scenario for supersymmetry breaking. This is a four parameter model, with one sign that must be chosen. There are many constraints from (lack of) data on e.g. the masses of the superpartners. The most restrictive constraint comes from the assumption that the LSP constitutes the dark matter. The cosmological dark matter density is known to better than $10 \%$ accuracy. In mSUGRA, the parameter region consistent with the dark matter density is very thin. Grid methods have a very difficult time finding these models, as the volume occupied by them in parameter space is a very small fraction of the total volume consistent with accelerator searches. Even with this relatively small number of parameters we find that MCMC is much superior to grid searches. Our future goal is to apply MCMC techniques to larger parameter spaces, with the expectation that only modest increases in computational resources will be required.

\section{Minimal Supergravity}

One of the most well studied frameworks for supersymmetry breaking is Minimal Supergravity [1]. This model distills the multitude of supersymmetry breaking parameters into merely four, plus one sign. It assumes that all sfermions share the same mass at the GUT scale, $m_{0}$. This contributes to the masses of the Higgs doublets as well. It assumes that the hypercharge, weak, and strong gauginos share the same mass at the GUT scale, $m_{1 / 2}$. There is a GUT scale trilinear coupling among scalars $A_{0}$. The last continuous parameter is taken to be the ratio of the Higgs vacuum expectation values (VEVs) at the weak scale, $\tan \beta$. Equivalently, a GUT scale boundary condition could be applied - this is the $B$ parameter. In this framework, the weak scale Higgs mass parameter $\mu^{2}$ is derived from the requirement of electroweak symmetry breaking, thus $\mu$ is derived up to a phase. To conserve CP, the phase of $\mu$ is chosen to be \pm 1 , the last parameter of the model. 
Many authors have studied the mSUGRA parameter space from many points of view [2]. In this paper we explore the full parameter space, subject to some basic constraints. The techniques described below allow us to vary all four mSUGRA parameters freely, while efficiently focusing on the interesting regions of parameter space. In contrast, studies in the literature typically illustrate several 2-parameter slices of the parameter space, usually holding $A_{0}$ and $\tan \beta$ fixed.

For each set of parameters, we compute the spectrum using ISAJET [3], and we compute other relevant quantities with DarkSUSY [4]. The model confronts current accelerator constraints on sparticle and Higgs masses [5], including preliminary results from LEP concerning a Standard Model-like Higgs [6] and the chargino mass [7]. Precision results for the $b \rightarrow s \gamma$ branching ratio from the CLEO and Belle collaborations are applied [8] (DarkSUSY computes the NLO $b \rightarrow s \gamma$ ). We mention here that the precise value of the mass of the top quark $m_{t}$ can have significant effects on mSUGRA models even within current error bars. PDG2002 quotes $m_{t}=174.3 \pm 5.1 \mathrm{GeV}$ [5], while a more recent evaluation from the CDF and D0 collaborations finds $m_{t}=178.0 \pm 4.3 \mathrm{GeV}$ [9]. In effect we need to take $m_{t}$ as one of the model parameters.

The anomalous magnetic moment of the muon $a_{\mu}=\left(g_{\mu}-2\right) / 2$, is sensitive to new physics such as supersymmetry. The experimental measurement has improved greatly in recent years [10], and there is a hint of a discrepancy with the Standard Model, though the theoretical calculations of the Standard Model hadronic contribution are somewhat in doubt $[11,12]$ due to a discrepancy between evaluations based on $e^{+} e^{-} \rightarrow$ hadrons and those based on hadronic decays of the $\tau$ lepton. Quoting the results of Davier et al. [11], the discrepancy is

$$
\begin{aligned}
& \Delta a_{\mu}=(27 \pm 10) \times 10^{-10}\left(e^{+} e^{-} \text {based }\right) \\
& \Delta a_{\mu}=(12 \pm 9) \times 10^{-10}(\tau \text { based })
\end{aligned}
$$

We apply the constraint that the SUSY contribution must be within $3 \sigma$ of one of these calculations,

$$
-15 \times 10^{-10}<\Delta a_{\mu}(\text { SUSY })<57 \times 10^{-10} .
$$

Furthermore, we will highlight models that fall within $1 \sigma$ of either of these measurements to simulate future constraints,

$$
3 \times 10^{-10}<\Delta a_{\mu}(\operatorname{SUSY}, 1 \sigma)<37 \times 10^{-10} .
$$

This latter constraint enforces that the supersymmetric correction be positive, ruling out $\mu<0$. Much of the power this constraint has is simply the requirement of positive $\mu$.

Of astrophysical interest, we compute the relic density of neutralinos in the model, as well as the neutralino - nucleon elastic scattering cross section. We are interested in models where the neutralino relic density is consistent with the cosmological dark matter density, as discussed in the next section. 


\section{Dark Matter}

We take the viewpoint that the observed density of cold dark matter is the most accurate measurement available indicating physics beyond the standard model [13]. The best single measurement to date is that of the Wilkinson Microwave Anisotropy Probe (WMAP) [14], which measured the angular power spectrum of the thermal fluctuations in the cosmic microwave background. These fluctuations encode information on many cosmological parameters, including the cosmological densities of many species relative to the critical density $\Omega_{X}=\rho_{X} / \rho_{c}$, where $\rho_{c}=3 H_{0}^{2} / 8 \pi G$, and $H_{0}$ is the Hubble constant. As is usually done, we take $H_{0}=100 h \mathrm{~km} \mathrm{~s}^{-1} \mathrm{Mpc}^{-1}$. The relevant parameters are then the total matter density $\Omega_{M} h^{2}$, the baryon density $\Omega_{B} h^{2}$ and the neutrino density $\Omega_{\nu} h^{2}$. The density of cold dark matter is then just $\Omega_{\mathrm{CDM}} h^{2}=\Omega_{M} h^{2}-\Omega_{B} h^{2}-\Omega_{\nu} h^{2}$. The WMAP results are as follows [14]

$$
\begin{aligned}
\Omega_{M} h^{2} & =0.135_{-0.009}^{+0.008} \\
\Omega_{B} h^{2} & =0.0224 \pm 0.0009 \\
\Omega_{\nu} h^{2} & <0.0072 \text { (95\% confidence). }
\end{aligned}
$$

Other cosmological probes, such as the Sloan Digital Sky Survey (SDSS) [15] give consistent and complementary results. We naively construct the cold dark matter density by adding the matter and baryon errors in quadrature, and simply expanding the lower error bar to accommodate the possibility of a neutrino component. Thus,

$$
\Omega_{\mathrm{CDM}} h^{2}=0.1126_{-0.013}^{+0.008} \text {. }
$$

We will consider the following range for the cold dark matter density, allowed at $95 \%$ confidence,

$$
0.087<\Omega_{\mathrm{CDM}} h^{2}<0.129 .
$$

In the next section, we require a likelihood function based on the relic density of neutralinos. We will use the WMAP likelihood function, assuming an asymmetric lognormal distribution.

$$
\begin{aligned}
\chi^{2} & =\left(\frac{\ln \left(\Omega_{\mathrm{CDM}} h^{2} / 0.113\right)}{\sigma}\right)^{2}, \\
\sigma & =0.068,\left(\Omega_{\mathrm{CDM}} h^{2}>0.113\right), \\
\sigma & =0.12,\left(\Omega_{\mathrm{CDM}} h^{2}<0.113\right) .
\end{aligned}
$$

The likelihood function is then determined from $\chi^{2}$ in the usual manner, $L=\exp \left(-\chi^{2} / 2\right)$.

\section{Markov Chain Monte Carlo}

The problem of efficiently scanning high-dimensional parameter spaces appears in most scientific disciplines. Clearly, direct grid scans can not be extended beyond a few dimensions. 
Markov Chain Monte Carlo (MCMC) algorithms were developed to firstly numerically find function minima, and secondly to explore the region "near" the minimum. The utility of these techniques for data analysis is obvious: most famously in cosmology the data from the WMAP satellite were analyzed in this way [16]. Detailed explanations may be found in Ref. [17].

A Markov chain is a sequence of points $P_{0} \rightarrow P_{1} \rightarrow P_{2} \rightarrow \ldots$, with repetitions allowed, together with a transition probability $W\left(P_{i+1} \mid P_{i}\right)$ from one point to the next. In a Markov Chain Monte Carlo, a Markov chain is constructed as follows. The first point of the chain $P_{0}$ is randomly chosen according to a prior probability $\Pi(P)$. A new point in the chain is proposed according to a proposal probability $G\left(P_{i+1} \mid P_{i}\right)$, and is accepted as part of the chain according to an acceptance probability $A\left(P_{i+1} \mid P_{i}\right)$. The transition probability is then $W\left(P_{i+1} \mid P_{i}\right)=G\left(P_{i+1} \mid P_{i}\right) A\left(P_{i+1} \mid P_{i}\right)$. It has been proven that if $L(P)$ is a probability distribution that satisfies the detailed balance condition $W\left(P_{j} \mid P_{i}\right) L\left(P_{i}\right)=W\left(P_{i} \mid P_{j}\right) L\left(P_{j}\right)$, then asymptotically, the points $P_{i}$ in the Markov chain defined by $W\left(P_{i+1} \mid P_{i}\right)$ are distributed according to the "equilibrium" distribution $L(P)$.

We use the Metropolis algorithm to construct chains of mSUGRA models, with the hope that the WMAP region will be strongly favored giving scans much more efficient than grid scans. A Markov Chain is constructed as follows. A random point $P_{0}$ in parameter space is chosen as the start of the chain, checking to see that it passes accelerator constraints. The likelihood function $L \equiv e^{-\chi^{2} / 2}$ is computed so that $L\left(P_{0}\right)=L_{0}$. From this point, a proposal step is made, to point $P_{p}$ with likelihood $L_{p}$. The Markov chain is advanced as follows. If $L_{p} \geq L_{0}$ take $P_{1}=P_{p}$, i.e., if the new point has a higher likelihood, take it. If $L_{p}<L_{0}$ take $P_{1}=P_{p}$ with probability $L_{p} / L_{0}$, otherwise take $P_{1}=P_{0}$, i.e., if the new point has a lower likelihood, take it with a probability equal to the ratio of likelihoods, otherwise take the new point equal to the old point. The process repeats from $P_{1}$, and the Markov chain is constructed. If the proposed point is better, the chain always advances there. If the proposed point is worse, the chain sometimes advances there - if it is only slightly worse, the chain will advance there most of the time, if it is much worse, the chain advances only rarely. This deceptively simple algorithm is very efficient at traversing a large-dimensional parameter space, in $O(N)$ time, in contrast to the $O\left(e^{N}\right)$ time for a grid search.

Of course the Metropolis algorithm for advancing the Markov chain is not the whole story. Choosing a proposal point based on the $i$-th point in the chain $P_{p}\left(P_{i}\right)$ in an optimal way is a subject of much research in the MCMC field. We now need to know something about the specific problem we are interested in. We would like to find the region of mSUGRA parameter space that is consistent with the WMAP data. This is a strange problem from the point of view of the usual applications of MCMC, namely we have many more model parameters (four) than data points (one). This is not a minimization problem at all, but one of finding the three-dimensional contours of relic density near the WMAP measured value. The degeneracies among parameters are exact. This contrasts the usual applications of MCMC, which involve many data points constraining a model with only a few parameters, with the goal of finding a best fit model and mapping the likelihood surface around it. 
In looking for an optimal strategy in this new context, we try several strategies for making proposal steps. The first issue involved is the question of how big a step should be taken. We can draw on a simple case for guidance. Take a likelihood function that is gaussian in the parameters (with zero means and unit widths) $L=\exp \left(-\sum x_{i}^{2} / 2\right.$ ), and take a proposal step gaussian about the current point, with width $N$ times the width of the likelihood function $P\left(p_{i}\right) \propto \exp \left(-\sum\left(p_{i}-x_{i}\right)^{2} /\left(2 N^{2}\right)\right)$. In the limit of a large number of parameters $D$, the probability to accept the proposed point is $P$ (accept) $=1-\operatorname{erf}(N \sqrt{D / 8})$. In this limit the optimal stepsize (maximizing $N^{2} P$ (accept), a measure of the "diffusion velocity" of the chain) is given by $N=2.381 / \sqrt{D}[18]$. This result assumes that the Markov chain is "burned in," namely it has found the region of reasonable likelihood. For even a few parameters these results are acceptable, though for one or two they break down, e.g. for one parameter, it is easy to show that the acceptance probability is $P($ accept $)=(2 / \pi) \tan ^{-1}(2 / N)$ and $N^{2} P$ (accept) has no maximum. ${ }^{1}$ However, it has been shown that $N \approx 2.4 / \sqrt{D}$ is a reasonable approximation even for $D=1,2$ [19]. What we can draw from this is an optimal acceptance probability, $P$ (accept) $=0.2338$. This means that the fastest exploration of the parameter space happens when only about $1 / 4$ of the proposals are accepted. This allows the stepsize to remain relatively large, but still a reasonable fraction of points are accepted. Based on this result, we apply a simple method for adapting the stepsize. If too many proposals are accepted in a row, it can be inferred that the probability to step is too large to optimally sample the parameter space. The indication is that the stepsize is too small and should be increased. Likewise, if too many proposals are rejected in a row, the acceptance probability may be too small and the stepsize may be too large. For example, based on an acceptance probability of $1 / 4$, three consecutive acceptances is already unlikely at the $98.4 \%$ level, and the stepsize should be increased. Aiming for a similar probability for the other extreme, if 14 proposals are rejected in a row, the stepsize should be decreased. In both cases we choose to change the stepsize by a factor of 2 . We have made crude explorations of these thresholds, and found that changing the stepsize for 3-4 consecutive acceptances or 6-8 consecutive rejections works fairly well. The exact numbers can be adjusted, but we find that the threshold for rejections should be higher than for acceptance. Equivalently, an acceptance probability less than 0.5 is ideal. Setting the threshold for rejections at twice the threshold for acceptances corresponds to an acceptance probability of $38 \%$.

The second issue we discuss is that of step direction. We know that there are exact degeneracies, as we place only one constraint on many parameters. We would like to step in a direction that is likely to find another good point. When the chain point is close to

\footnotetext{
${ }^{1}$ We have derived an exact expression for $\mathrm{P}$ (accept) as a function of $N$ and $D$. In full generality the expression is a double integral, not obviously tractable for $D>1$. In the limit of large $D$ the integrand simplifies, and we reproduce the limit given in Ref. [18]. For $D \geq 2$ the result is

$$
P(\text { accept })=\frac{2^{5 / 2-D} N^{1-D}}{\Gamma\left(\frac{D}{2}\right) \Gamma\left(\frac{D-1}{2}\right)} \int_{0}^{\infty} d x x^{D-1} e^{-x^{2} / 2} \int_{0}^{x} d y y^{D-2} e^{-y^{2} / 2 N^{2}} \sum_{ \pm} \operatorname{erf}\left(\frac{\sqrt{x^{2}-y^{2}} \pm x}{N \sqrt{2}}\right) .
$$

For $D=2$ we find again that the function $N^{2} P($ accept $)$ has no maximum, in fact for $N \gg 1, P($ accept $) \propto N^{-D}$.
} 
the WMAP value of $\Omega_{\mathrm{CDM}} h^{2}$, we would like the next point to lie (approximately) on the $\Omega_{\mathrm{CDM}}=\Omega_{\mathrm{CDM}, \mathrm{WMAP}}$ surface. The most obvious thing to do is to calculate the likelihood gradient at the current point in the Markov chain, and step orthogonally to the gradient. We do this when 4 (or the number of dimensions) consecutive rejections are collected, as

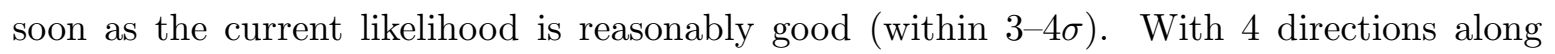
which the difference in likelihood is known, the gradient vector can be computed. ${ }^{2}$ After this, steps are taken in arbitrary directions perpendicular to the gradient vector. A refinement of this procedure is to allow a small step along the gradient, though much smaller than that perpendicular. This can be done in a fixed manner (e.g. 10:1 ratio, 20:1 ratio), or adaptively. We employ the gradient technique for most of the mSUGRA scans discussed in this paper. We remark here that this choice of proposal probability depends through the gradient and stepsize on more than just the previous point in the Markov chain. As a consequence, its associated transition probability does not satisfy the condition of detailed balance, and we cannot immediately prove that our gradient algorithm converges to the likelihood distribution $L(P)$. Nevertheless, based on the intuition that an "equilibrium" distribution should not depend on the initial conditions or on the way equilibrium is approached, we expect that the points in the Markov chain are distributed according to the likelihood $L(P)$ after the chain has "converged." It must however be kept in mind that the final distribution of points in parameter space depends also on where we choose the initial point of each chain, i.e. our prior distribution.

Another method for using directional information involves the interactions among several Markov chains. The basic assumption is that if two Markov chains have found good regions, then the line connecting their current positions might also be in the good region. For each chain, a random second chain is selected, and the step taken is in the direction (positive or negative) of this chain. This is the "snooker" algorithm. A few of our scans use this algorithm, trying interactions between 8 chains, or 128 chains. We find neither does a great job of covering the full surface, and that perhaps more chains are required. This difficulty is possibly related to the fact that we are trying to trace a surface instead of finding a global minimum.

\section{Scanning the mSUGRA Parameter Space}

We now explore the mSUGRA parameter space using the techniques of the previous section. First, we employ a very crude grid search, taking only a few points along the $\tan \beta$ and $A_{0}$ axes. Roughly $1 \%$ of the scanned points pass the WMAP $2 \sigma$ relic density cut. Crudely, this is the 4-dimensional "efficiency" of the grid search.

As the first illustration of the MCMC method, we attempt to duplicate a typical mSUGRA scan from the literature. We choose Fig. 3 of Ref.[20], which illustrates the usual basic fea-

\footnotetext{
${ }^{2}$ We compute the gradient of $\Omega_{\mathrm{CDM}} h^{2}$ rather than the gradient of the likelihood function. The two gradients share the same direction since our likelihood is a function of $\Omega_{\mathrm{CDM}} h^{2}$ only. However, the gradient of the likelihood function vanishes on the WMAP surface, thus we use the former gradient.
} 
tures - at moderate $\tan \beta$, acceptable relic density occurs only for the stau coannihilation region at small $m_{0}$ or the focus point region at large $m_{0}$ where chargino coannihilations are important. This model has $A_{0}=0, \tan \beta=30, \mu>0$, and $m_{t}=175 \mathrm{GeV}$. Fixing these parameters, we make a 2 dimensional scan in $m_{0}$ and $m_{1 / 2}$. In Fig. 1 we plot every point where the relic density was calculated. Points that pass the WMAP relic density cut are highlighted in red, and those with relic density below the WMAP region are highlighted in blue. It is clear that the MCMC technique does an excellent job of automatically finding the WMAP preferred region. For these scans, the gradient method was used. The usual features are clear in that the acceptable regions are the stau coannihilation funnel at small $m_{0}$ and the focus point region at large $m_{0}$. About 40,000 points have been generated in this scan. Notice that only a small fraction of points lies outside the good WMAP region, so few that they can be counted individually on the figure. This indicates that the Markov chains converge to the good region rapidly.

We now extend the scans to the full 4 dimensional parameter space. We are searching for the 3 dimensional surface near which the relic density is acceptable. We separately scan both signs of $\mu$, and take three values for the top quark mass: $m_{t}=174.3 \mathrm{GeV}$ (PDG 2002 central value [5]), $m_{t}=178.0 \mathrm{GeV}$ (current $\mathrm{CDF} / \mathrm{D} 0$ central value [9]), and $m_{t}=181.7$ $\mathrm{GeV}$ (within $1 \sigma$ of the central value). Using the MCMC techniques of the previous section, predominantly the gradient method, but also with some scans using the snooker algorithm, we find efficiencies of 20\%-30\% to find points that pass the relic density cut. This is remarkable in that the acceptable region is found automatically. In total, we have sampled some 2.4 million models, with more than 500 thousand within the WMAP region. The full extent of the scans is as follows,

$$
\begin{gathered}
50 \mathrm{GeV}<m_{0}<50 \mathrm{TeV} \\
50 \mathrm{GeV}<m_{1 / 2}<20 \mathrm{TeV} \\
-20 \mathrm{TeV}<A_{0}<20 \mathrm{TeV} \\
2<\tan \beta<60 .
\end{gathered}
$$

MCMC techniques allow us to scan freely in all 4 parameters. Since the MCMC algorithm is essentially linear in the number of parameters, the utility of the technique for more complex models is clear.

As we are scanning freely in $A_{0}$, we must be careful about unacceptable vacua, both potential directions unbounded from below (UFB) and the so-called charge and/or color breaking (CCB) vacua that occur if e.g. the stop gets a VEV [21]. We apply a simple constraint [22] that (conservatively) allows for CCB minima as long as they are long-lived,

$$
A_{t}^{2}+3 \mu^{2}<7.5\left(m_{\tilde{t}_{L}}^{2}+m_{\tilde{t}_{R}}^{2}\right)
$$

and the parameters are taken at the weak scale. This constraint in particular excludes the stop from getting a VEV, removing a small number of models where $\left|A_{0}\right| \gg m_{0}$. Even this 
constraint may not be permissive enough; recent work indicates that radiative corrections soften some of these constraints considerably [23].

\section{Projections of mSUGRA Parameter Space}

After performing these scans, we have a 4 dimensional cloud of models, a significant fraction of which pass the WMAP relic density cut. In order to display it easily, we project onto the six two-dimensional coordinate planes. We will illustrate both theoretical (involving the 4 mSUGRA parameters) and phenomenological (e.g. LSP mass and cross section) examples.

As an application to dark matter searches, we consider the spin-independent neutralinonucleon elastic scattering cross section, $\sigma_{\mathrm{SI}}$. We plot the neutralino mass $m_{\chi}$ vs. $\sigma_{\mathrm{SI}}$ in Fig. 2, for every model passing the WMAP constraint. We illustrate the DAMA preferred region [24], current experimental bounds from EDELWEISS [25] and CDMS [26], and the future reach of several experiments, running (CDMS II [27], CRESST II [28]) and proposed (GENIUS [29], CryoArray [30] and XENON [31]). For an extensive collection of data and projections for dark matter experiments see Ref. [32]. We have highlighted models having a $\Delta a_{\mu}$ (SUSY) within $1 \sigma$ of the current experimental bounds to illustrate the correlation between $a_{\mu}$ and $\sigma_{\mathrm{SI}}$ [33].

In Fig. 3 and Fig. 4, we plot all six 2-dimensional projections of the 4 dimensional mSUGRA parameter space, with one subplot each for $\mu= \pm 1$. There is one plot for every parameter pair in $m_{0}, m_{1 / 2}, A_{0}$ and $\tan \beta$. Fig. 3 illustrates only those models that obey the WMAP constraint, while Fig. 4 illustrates all models passing the accelerator constraints. Seen in projection, the WMAP constraint does not look very powerful, but it typically singles out very thin surfaces as seen in Fig. 1. These thin surfaces disappear in projection, since their exact position in a 2-dimensional projection is a function of the other two parameters.

\section{Discussion}

We have shown that MCMC is a powerful technique for scanning high dimensional parameter spaces in supersymmetry by demonstrating that it can navigate the mSUGRA parameter space and find regions with relic density acceptable to WMAP. This is a heartening conclusion. On the face of it, finding acceptable mSUGRA models is a very difficult problem for any function minimization technique. There are several issues involved. First, there are exact degeneracies. What we really need is a contouring algorithm. The gradient method alleviates this difficulty. Second, the acceptable regions tend to be on the edges of the allowed parameter space. Third, there are acceptable regions that are disjoint, or nearly so. A certain amount of brute force is required; what MCMC allows for is a considerable reduction in the amount of brute force necessary.

A more correct approach to the parameter scanning would use likelihoods based on ac-

celerator data rather than hard constraints. In particular, the $b \rightarrow s \gamma$ branching ratio and 
$\Delta a_{\mu}$ (SUSY) would be easy to implement in this way. Limits on particle masses could be expressed as likelihoods as well, though most values would be equally likely, with the likelihood dropping as the "limit" was approached. In fact, an analysis of the $\chi^{2}$ fit for the combination $b \rightarrow s \gamma, \Delta a_{\mu}$ (SUSY) and $\Omega_{\mathrm{CDM}} h^{2}$ has been performed [34]. An obvious extension of our work would be to use this likelihood function with three data points to do an MCMC scan of parameter space as we have done. However, none of the current accelerator constraints is nearly as powerful as the relic density constraint. From the viewpoint of finding acceptable models, we have used the most important constraint in the MCMC scans.

The likelihood function applied need not be related to real data. To scan an interesting region in parameter space, a likelihood function could be designed to favor that region. For example, in addition to including the WMAP relic density measurement, a likelihood function could be constructed to favor large elastic scattering cross sections, or any other interesting signal. In this way, models consistent with the WMAP relic density AND with high cross sections would be preferentially chosen. We leave explorations of this possibility to future work. Optimistically, if supersymmetry is discovered in e.g. LHC data, new terms in the likelihood function would be required. The gradient method can be adapted when the number of terms is less than the number of parameters: the gradient of each constraint is computed in turn, and steps are taken in the parameter subspace orthogonal to all such gradients.

It is tempting to ascribe a statistical significance to the density of models calculated (more correctly, using the points of the Markov Chain which excludes rejected points and possibly includes accepted points multiple times). We urge caution here, simply stating that the higher density regions tend to be associated with acceptable relic density and as such are more likely.

In a sense (not terribly well-defined), mSUGRA is disfavored by the WMAP results in that getting a reasonable relic density is "difficult," usually requiring coannihilations or strong resonance effects. We plan to explore more general model frameworks which may relax these difficulties. MCMC will allow such studies, while not requiring particularly immense computational resources.

\section{Acknowledgments}

E. A. B. thanks Phil Marshall and John Peterson for selflessly educating him on MCMC techniques, and thanks Michael Peskin for many useful conversations on mSUGRA models. We thank the Michigan Center for Theoretical Physics for hospitality during the Dark Side of the Universe workshop, where some of this research was done. This work was supported in part by the U.S. Department of Energy under contract number DE-AC03-76SF00515.

\section{References}

[1] A. Chamseddine, R. Arnowitt and P. Nath, Phys. Rev. Lett. 49 (1982) 970; R. Barbieri, S. Ferrara and C. Savoy, Phys. Lett. B 119 (1982) 343; N. Ohta, Prog. Theor. Phys. 70 (1983) 542; L. J. Hall, J. Lykken and S. Weinberg, Phys. Rev. D 27 (1983) 2359. 
[2] For a representative sample of recent papers studying mSUGRA see e.g. J. Ellis, T. Falk and K. Olive, Phys. Lett. B 444 (1998) 367; J. Ellis, T. Falk, K. Olive and M. Srednicki, Astropart. Phys. 13 (2000) 181; M.E. Gómez, G. Lazarides and C. Pallis, Phys. Rev. D 61 (2000) 123512 and Phys. Lett. B 487 (2000) 313; K. L. Chan, U. Chattopadhyay and P. Nath, Phys. Rev. D 58 (1998) 096004; J. Feng, K. Matchev and T. Moroi, Phys. Rev. Lett. 84 (2000) 2322 and Phys. Rev. D 61 (2000) 075005; J. Feng, K. Matchev and F. Wilczek, Phys. Lett. B 482 (2000) 388 and Phys. Rev. D 63 (2001) 045024; H. Baer and M. Brhlik, Phys. Rev. D 57 (1998) 567; M. Drees and M. Nojiri, Phys. Rev. D 47 (1993) 376; H. Baer and M. Brhlik, Phys. Rev. D 53 (1996) 597; H. Baer, M. Brhlik, M. Diaz, J. Ferrandis, P. Mercadante, P. Quintana and X. Tata, Phys. Rev. D 63 (2001) 015007; J. Ellis, T. Falk, G. Ganis, K. Olive and M. Srednicki, Phys. Lett. B 510 (2001) 236; L. Roszkowski, R. Ruiz de Austri and T. Nihei, J. High Energy Phys. 0108 (2001) 024; A. Bottino, F. Donato, N. Fornengo and S. Scopel, Phys. Rev. D 63 (2001) 125003; A. Lahanas and V. Spanos, Eur. Phys. J. C 23 (2002) 185; H. Baer, C. Balazs, A. Belyaev, J. Mizukoshi, X. Tata and Y. Wang, J. High Energy Phys. 0207 (2002) 050; H. Baer, T. Krupovnickas and X. Tata, J. High Energy Phys. 0307 (2003) 020; H. Baer, C. Balazs, A. Belyaev, T. Krupovnickas and X. Tata, J. High Energy Phys. 0306 (2003) 054; H. Baer, A. Belyaev, T. Krupovnickas and X. Tata, J. High Energy Phys. 0402 (2004) 007; M. Battaglia, A. De Roeck, J. R. Ellis, F. Gianotti, K. A. Olive and L. Pape, Eur. Phys. J. C 33 (2004) 273; J. Edsjö, M. Schelke, P. Ullio and P. Gondolo, J. Cosm. Astropart. Phys. 0304 (2003) 001; J. Edsjö, M. Schelke and P. Ullio, astro-ph/0405414.

[3] H. Baer, F. E. Paige, S. D. Protopescu and X. Tata, hep-ph/0312045.

[4] P. Gondolo, J. Edsjö, L. Bergström, P. Ullio and E. A. Baltz, astro-ph/0012234; P. Gondolo, J. Edsjö, P. Ullio, L. Bergström, M. Schelke and E. A. Baltz, astro-ph/0211238, J. Cosm. Astropart. Phys., in press, (astro-ph/0406204); http://www.physto.se/ ^edsjo/darksusy/.

[5] K. Hagiwara et al. (Particle Data Group), Phys. Rev. D 66 (2002) 010001.

[6] LEP Higgs Working Group, note CERN-EP-2003-011 (http://lephiggs .web.cern.ch/LEPHIGGS/papers/index.html).

[7] LEP SUSY Working Group, ALEPH, DELPHI, L3 and OPAL experiments, notes LEPSUSYWG/01-03.1 and LEPSUSYWG/02-04.1 (http://lepsusy.web.cern.ch/lepsusy/Welcome.html).

[8] S. Chen et al. (Cleo Collaboration), Phys. Rev. Lett. 87 (2001) 251807; K. Abe et al. (Belle Collaboration), Phys. Lett. B 511 (2001) 151.

[9] CDF Collaboration, D0 Collaboration and Tevatron Electroeak Working Group (http://tevewwg.fnal.gov), hep-ex/0404010.

[10] H. N. Brown, et al., Phys. Rev. Lett. 86 (2001) 2227; G. W. Bennett, et al., Phys. Rev. Lett. 89 (2002) 101804, Phys. Rev. Lett. 92 (2004) 161802.

[11] M. Davier, S. Eidelman, A. Höcker and Z. Zhang, Eur. Phys. J. C 27 (2003) 497, Eur. Phys. J. C 31 (2003) 503.

[12] K. Hagiwara, A. D. Martin, D. Nomura and T. Teubner, Phys. Lett. B 557 (2003) 69, Phys. Rev. D 69 (2004) 093003. 
[13] For reviews of supersymmetric dark matter see e.g. G. Jungman, M. Kamionkowski and K. Griest, Phys. Rept. 267 (1996) 195; L. Bergström, Rept. Prog. Phys. 63 (2000) 793.

[14] D. N. Spergel et al., Astrophys. J. Suppl. 148 (2003) 175.

[15] M. Tegmark et al., Phys. Rev. D 69 (2004) 103501.

[16] L. Verde et al., Astrophys. J. Suppl. 148 (2003) 195.

[17] W. R. Gilks, S. Richardson and D. J. Spiegelhalter, Markov Chain Monte Carlo in Practice, Chapman \& Hall, London, (1996).

[18] G. O. Roberts, A. Gelman and W. R. Gilks, Ann. Appl. Prob. 7 (1997) 110.

[19] J. Dunkley, M. Bucher, P. G. Ferreira, K. Moodley and C. Skordis, astro-ph/0405462.

[20] H. Baer, A. Belyaev, T. Krupovnickas and J. O'Farrill, hep-ph/0405210

[21] For example see J. F. Gunion, H. E. Haber and M. Sher, Nucl. Phys. B 306 (1988) 1; J. A. Casas, A. Lleyda and C. Muñoz, Nucl. Phys. B 471 (1996) 3; D. G. Cerdeño, E. Gabrielli, M. E. Gomez and C. Muñoz, J. High Energy Phys. 0306 (2003) 030.

[22] A. Kusenko, P. Langacker and G. Segrè, Phys. Rev. D 54 (1996) 5824.

[23] P. M. Ferreira, hep-ph/0406234.

[24] R. Bernabei et al., Riv. Nuovo Cim. 26 (2003) 1.

[25] A. Benoit et al., Phys. Lett. B 545 (2002) 43.

[26] D. S. Akerib et al., astro-ph/0405033.

[27] http://cdms.berkeley.edu/

[28] http://wwwvms.mppmu.mpg.de/cresst/

[29] H. V. Klapdor-Kleingrothaus, in "Beyond the desert 1997," Castle Ringberg, Germany, eds. H. V. Klapdor-Kleingrothaus and H. Paes (IOP, Bristol, 1998), p. 485; H. V. Klapdor-Kleingrothaus et al., in "Beyond the desert 1999," Castle Ringberg, Germany, eds. H. V. Klapdor-Kleingrothaus and I. Krivosheina (IOP, 2000), p. 915.

[30] R. W. Schnee, D. S. Akerib and R. J. Gaitskell, astro-ph/0208326.

[31] E. Aprile et al., in Proceedings of Xenon '01, astro-ph/0207670, http://www . astro. columbia.edu/ ${ }^{1 x e / X E N O N / . ~}$

[32] R. J. Gaitskell and V. Mandic, http://dmtools.berkeley.edu/limitplots/

[33] M. Drees, Y. G. Kim, T. Kobayashi and M. M. Nojiri, Phys. Rev. D 63 (2001) 115009;

E. A. Baltz and P. Gondolo, Phys. Rev. Lett. 86 (2001) 5004, Phys. Rev. D 67 (2003) 063503.

[34] H. Baer and C. Balazs, J. Cosm. Astropart. Phys. 0305 (2003) 006. 


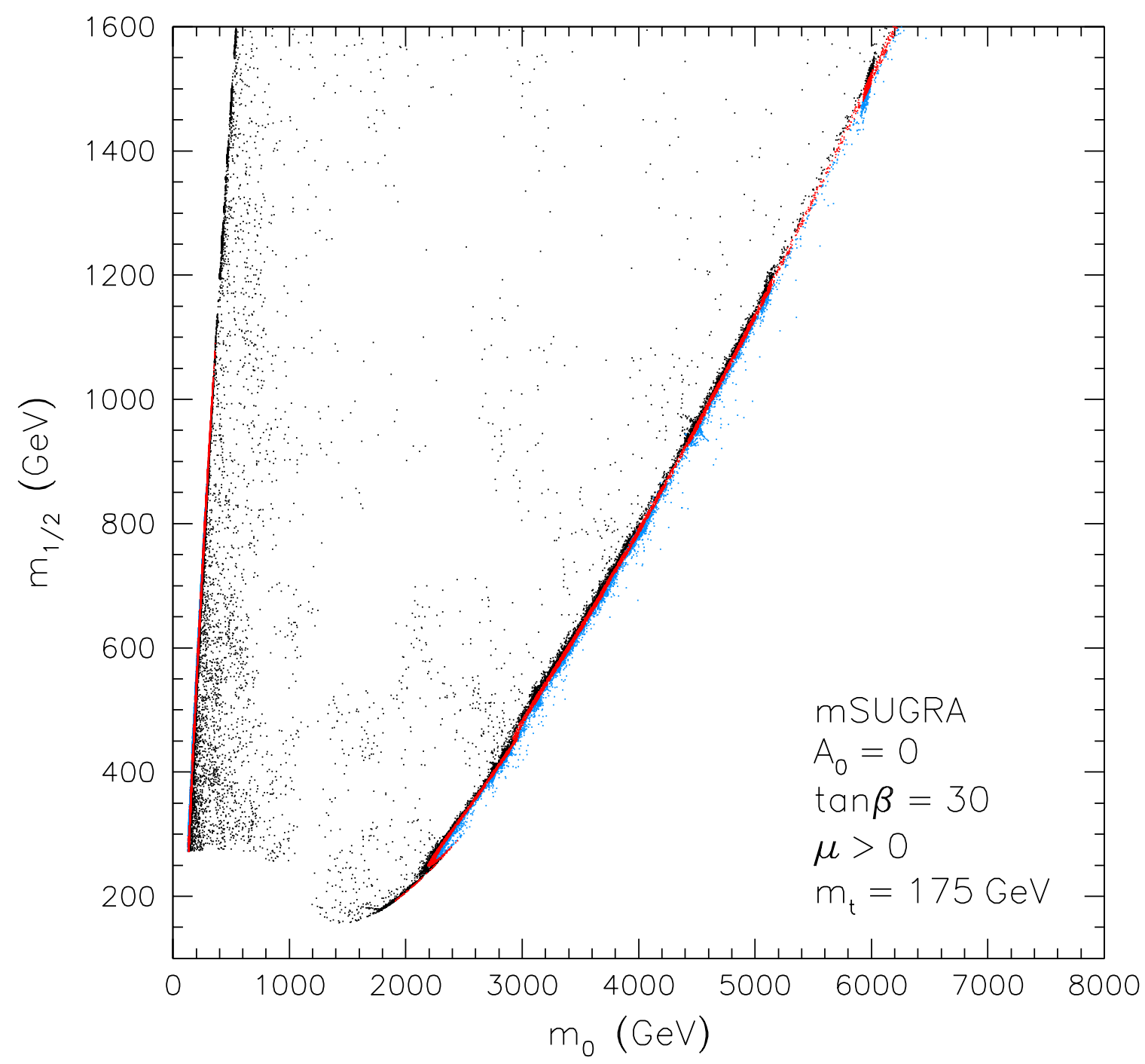

Figure 1: MCMC scan in $m_{0}$ and $m_{1 / 2}$, holding other parameters fixed. All points where the relic density was calculated are included. Compare with Fig. 3 of Ref. [20]. The strong preference for "interesting" regions is clear. Black points have $\Omega_{\chi} h^{2}$ more than $2 \sigma$ too large according to the WMAP constraint, red points are within the $2 \sigma$ region, and blue points have too little relic density at the $2 \sigma$ level - perfectly acceptable, but not as the sole constituent of dark matter. The gradient method was used for these scans. For some chains, the gradient estimate was not very good, e.g. the feature at $m_{0}=6 \mathrm{TeV}, m_{1 / 2}=1.5 \mathrm{TeV}$. These runs allow steps along the gradient of $5 \%$ or $10 \%$ of the step length. This plot is meant to illustrate the fact that very little time is spent exploring "uninteresting" regions of the parameter space. About 40,000 models are shown here, equivalent to a 200x200 grid search. For two dimensions, MCMC may not be a big improvement, but in more than two dimensions, it gains a clear advantage. Note that since we include all points, these are NOT the Markov Chains. The Markov Chains exclude rejected points and include some points multiple times. The Markov chains do not however reflect the computational efficiency that is a major concern in this work. 


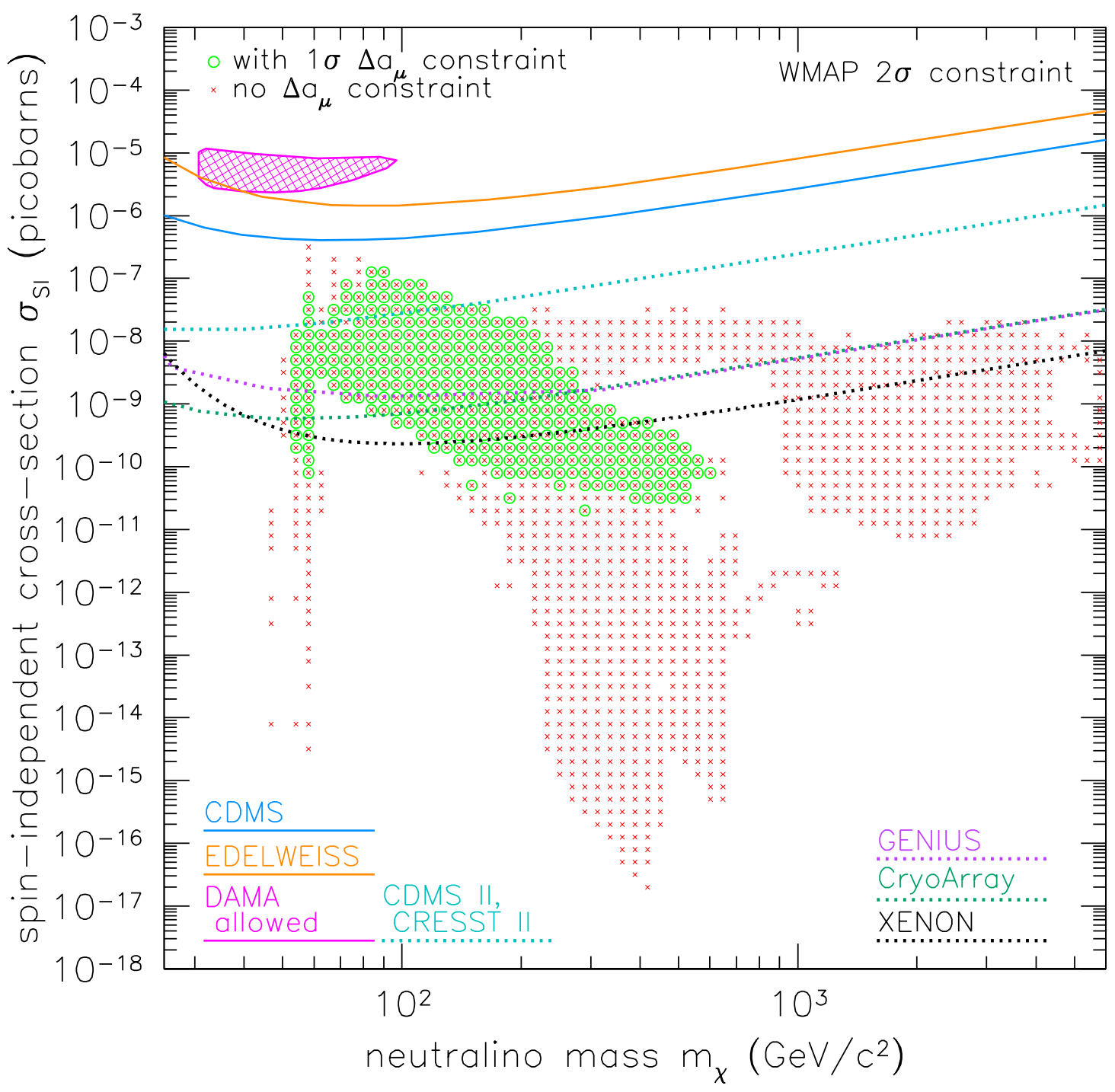

Figure 2: Spin-independent elastic scattering cross section of neutralinos on protons. The models have been collected in bins in a square grid. Current experimental limits from EDELWEISS and CDMS II are shown, as well as the region favored by the DAMA annual modulation result. The proposed reach of the CDMS II, CRESST II, CryoArray, GENIUS, and XENON experiments is also shown. Models falling withing $1 \sigma$ of the current measurement of $a_{\mu}$ are highlighted by green circles, though not all models in those bins necessarily pass the $a_{\mu}$ cut. 


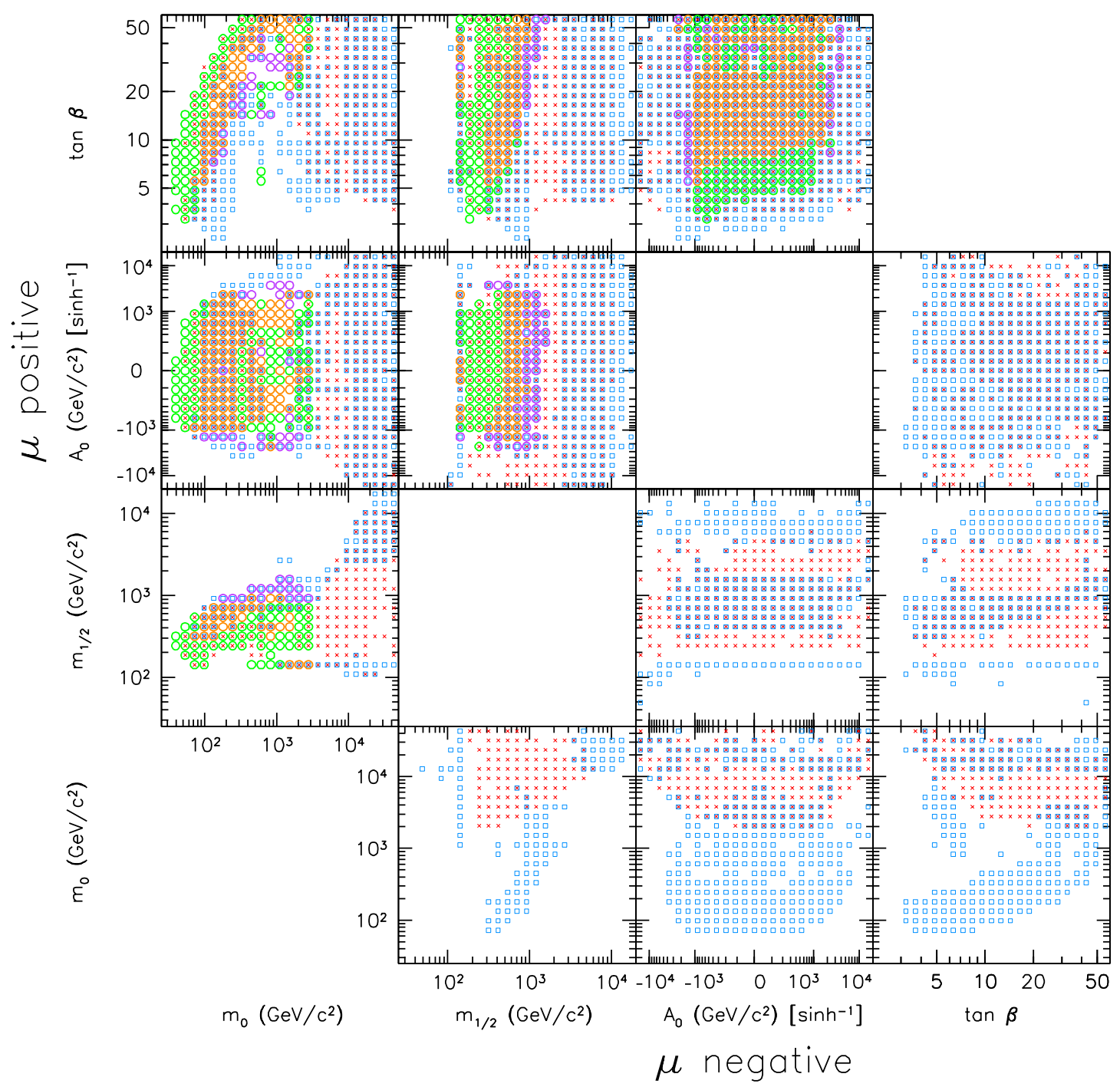

Figure 3: Projections of mSUGRA parameter space obeying the WMAP constraint on relic density. All six 2-dimensional projections of the four continuous parameters $m_{0}, m_{1 / 2}, A_{0}$ and $\tan \beta$ are illustrated, with $\mu$ positive in the upper left and $\mu$ negative in the lower right. The green, orange and purple circles indicate models within $1 \sigma$ of the current measurement of the muon $a_{\mu}$. Green indicates that all models passing the $a_{\mu}$ cut will be detectable by the proposed XENON experiment, orange indicates that some such models will be detectable, and purple indicates that no such models will be detectable. The red crosses show models that fail the $a_{\mu}$ constraint, and will be detectable by XENON, and the blue squares show models that fail the $a_{\mu}$ constraint, and will not be detectable. In projection, the WMAP constraint does not seem to be very powerful, in contrast to Fig. 1. This is simply because the thin sheet of allowed parameter space is not perpendicular to any particular one of the mSUGRA parameters. Note that $m_{0}, m_{1 / 2}$, and $\tan \beta$ are plotted logarithmically, while $A_{0}$ is plotted as $\sinh ^{-1}\left(A_{0} / 100 \mathrm{GeV}\right)$, with the first non-zero tickmark being $100 \mathrm{GeV}$. 


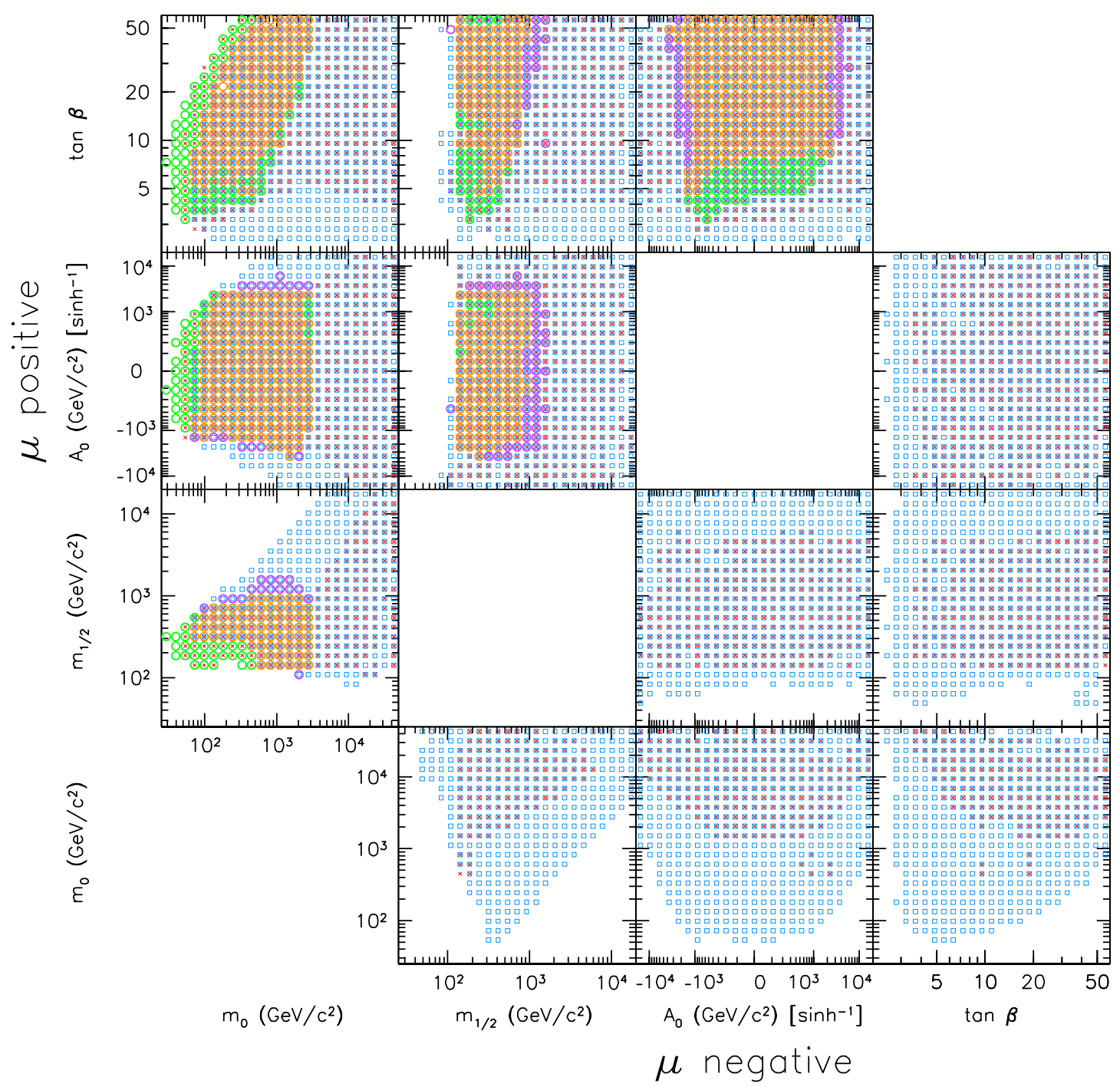

Figure 4: Projections of mSUGRA parameter space without the WMAP constraint on relic density. The points are plotted as in Fig. 3. The WMAP constraint does not appear to be making a huge difference when viewed in projection, though in fact the 4-dimensional volume occupied by acceptable models is quite small. 